\title{
An improved empirical dynamic control system model of global mean sea level rise and surface temperature change
}

\author{
Qing $\mathrm{Wu}^{1}$ • Quang-Hung Luu ${ }^{2,3} \cdot$ Pavel Tkalich $^{2} \cdot$ Ge Chen $^{1,4}$
}

Received: 17 May 2016 / Accepted: 12 January 2017 / Published online: 13 March 2017

(C) The Author(s) 2017. This article is published with open access at Springerlink.com

\begin{abstract}
Having great impacts on human lives, global warming and associated sea level rise are believed to be strongly linked to anthropogenic causes. Statistical approach offers a simple and yet conceptually verifiable combination of remotely connected climate variables and indices, including sea level and surface temperature. We propose an improved statistical reconstruction model based on the empirical dynamic control system by taking into account the climate variability and deriving parameters from Monte Carlo cross-validation random experiments. For the historic data from 1880 to 2001, we yielded higher correlation results compared to those from other dynamic empirical models. The averaged root mean square errors are reduced in both reconstructed fields, namely, the global mean surface temperature (by 24-37\%) and the global mean sea level (by $5-25 \%$ ). Our model is also more robust as it notably diminished the unstable problem associated with varying initial values. Such results suggest that the model not only enhances significantly the global mean reconstructions of temperature and sea level but also may have a potential to improve future projections.
\end{abstract}

Ge Chen

gechen@ouc.edu.cn

1 Qingdao Collaborative Innovation Center of Marine Science and Technology, College of Information Science and Engineering, Ocean University of China, Qingdao 266100, China

2 Tropical Marine Science Institute, National University of Singapore, 18 Kent Ridge Road, Singapore 119227, Singapore

3 College of Science, Vietnam National University, Hanoi, 334 Nguyen Trai Street, Hanoi, Vietnam

4 Laboratory for Regional Oceanography and Numerical Modeling, Qingdao National Laboratory for Marine Science and Technology, Qingdao 266100, China

\section{Introduction}

Substantial traces from oceans, atmosphere, and inland observations all result in the interpretation: climate has been changing drastically since the beginning of the twentieth century. Due to the accumulation of greenhouse gases, the global surface temperature was reported to rise about $0.85{ }^{\circ} \mathrm{C}$ since 1880 , and sea level rise is one of its apparent consequences. Centennial increases in both temperature and sea level cause great impacts on the ecosystem and human lives, especially in coastal areas where majority of global population reside. Using the empirical orthogonal functions (EOF) method, Church and White (2011) reconstructed the global mean sea level since 1900 to yield a rising trend of $1.7 \mathrm{~mm} / \mathrm{year}$. Applied statistical techniques, recently Hay et al. (2015) derived a slightly slower rate, $1.2 \mathrm{~mm} /$ year. Due to committed warming, the upward trends are projected to be continued at least in the next half century even if the anthropogenic emissions are reduced. Quantifying the changes is thus challenging and crucial to better assess and mitigate its global impacts.

In the climate interactive process, the increase in temperature influences the rise in sea level through three major mechanisms: the thermal expansions of oceanic waters due to their absorption of heats, the melting of ice sheets and glaciers at the Earth's poles due to the warming, and the alternation of inland hydrological and glacial sources. The underlying phenomena are complex within different time scales, leaving large uncertainties in linking sea level rise to the temperature change. For instance, our understanding of the melting rate of ice sheets is still poor, in spite of their potential largest contribution in the case of complete melting (up to $70 \mathrm{~m}$ according to IPCC (2013)).

Physically based climate and ocean models have gained notable successes in estimating of sea level response to given temperature scenarios (IPCC 2013). Numerical models are 
run at high resolutions, coupled with climatic and oceanic forcings to reproduce the temporal state of sea levels. However, the accuracy in presenting long-term variability of global mean sea level from their models are constrained by demands of huge computational resources, model's parameterizations, and presently, humble understanding of physical contributors to the system such as the abovementioned ice melt process and modeling complexity issues (Rahmstorf 2007; Schmith et al. 2012; Aral et al. 2012; Chang et al. 2014).

As an attempt to fill the gap left by the physically based models, the statistical approach was presented with the purpose of restoration for the state of sea level using its own past responses. To reconstruct the historic global mean sea levels, its strategy is to extract the eigenvalue matrices (i.e., principal components) of sea level data from satellite altimeter, and then combine them with temporal changes from tide gauge records (e.g., Church and White 2011; Hay et al. 2015). For the first time, the variability and long-term trend of global sea level change have been reasonably recognized (IPCC 2007; IPCC 2013) with aforementioned values. Nevertheless, since sea level change is modulated by various external nonlinear physical factors, mathematically (i.e., blindly) extracting the linear eigenvalues incongruously introduces bias into the estimates of rate of trend and magnitude of variability.

Based on such reconstructed datasets, the empirical approach is developed with the major emphasis on estimating sea level change in association with other physical variables. As the result, its distinct advantage is the capability to project future change of sea level with respect to different climate scenarios, as long as the relationship between sea level and physical variables such as surface temperature are elucidated. On the other hand, this approach produces additional dimension to assess the sea level change associated with climate projections computed by physics-based numerical models.

Synoptically, empirical models are classified into two categories: the semi-empirical approach and the dynamic statistical method. The first semi-empirical model proposed by Rahmstorf (2007) is based on the assumption that a linear relationship exists between the rate of sea level change $(d H /$ $d t)$ and the change of temperature $(T)$ with respect to a base temperature $T_{0}$. This approach gained notable successes for its simplicity and skill in reconstructing the historic global sea level data. The estimated rate of sea level rise from this model is about 3-4 mm/year from 1993 to 2006, which is similar to the observed rate of $3.3 \mathrm{~mm} /$ year from satellite altimetry and much better than the rate of $1.9 \mathrm{~mm} /$ year produced by numerical models (Jevrejeva et al. 2012). To improve the work of Rahmstorf (2007), Vermeer and Rahmstorf (2009) developed a modified dual model by incorporating the rapid response of temperature change into the model. On the other hand, Jevrejeva et al. (2009) used a similar model to successfully prove that before 1800, natural forcing (including volcano eruptions and solar radiation) is the main driver of sea level change; while thereafter, the majority $(75 \%)$ contributions to the rise are anthropogenic factors (related to greenhouse gases and aerosols).

In the dynamic statistical approach, it is argued that the changes of sea level and temperature are not independent but correlated in a feedback mechanism. Inspired by this idea, Aral et al. (2012) established a dynamic system model (DSM) describing the changes of the sea level and temperature in an interactive form. They pointed out that the rates of sea level rise and temperature change are functions of the temporal states of temperature and sea level, which can be derived from the proportional relationship with high correlations. At the same time, Schmith et al. (2012) adopted the dynamic concept by applying the vector error correction (VEC) model which is commonly used in econometric research to describe the relationship between sea level and temperature. They highlighted that the temperature change is casually related to sea level due to a large ocean heat capacity, which further confirmed the fundamental feedback hypothesis described by Aral et al. (2012).

Among the empirical approaches, the dynamic models have promisingly enabled us to link the global sea level rise with the temperature change. At one hand, it considers the changes of both sea level and temperature in an interactive system which is more intuitive and complete than the semiempirical approach. On the other hand, the dynamic method permits an extra capability on estimating temperature change. While the semi-empirical model requires the update of temperature at each step, the DSM is able to run seamlessly with the initial value once parameters are calibrated. Further, Guan et al. (2013) formulated a dynamic control system model (DCSM) in an attempt to introduce the anthropogenic impact as a control variable in temperature. However, the model is unable to reduce large errors in reconstructing temperature, especially during the early twentieth century warming.

The remaining residuals in sea level and temperature have been associated with other external forcings. In fact, previous studies suggested that natural influence including radiative forcing and volcano eruption events can play a critical role in driving climate change (Church et al. 2005; Trenberth and Fasullo 2013). While radiative forcing could not solely resolve such residuals (Andronova and Schlesinger 2000), fluctuations of global mean sea level and temperature have been tightly related to climate variability over the interannual to interdecadal time scales (IPCC 2013). For instance, the El Niño-Southern Oscillation (ENSO) and the Atlantic Multidecadal Oscillation (AMO) have been known to have a great impact on the temperature and sea level variability (e.g., Kravtsov and Spannagle 2008; Falvey and Garreaud 2009; Dong and Zhou 2014; Zhang and Church 2012; Cazenave et al. 2014; Luu and Tkalich 2014). Simply regard them as the noises that could potentially lead to a biased estimation of changing rates and neglect the significant contribution of their 
effects which eventually cause a larger uncertainty in future projections. Thus, it is necessary to account for the climate variability in the estimation of changes in global mean sea level and surface temperature.

In this study, we proposed an alternative modification to the DCSM by taking the climate variability into account, and then improve the novel estimation of parameters through applying the Monte Carlo cross-validation technique. The structure of paper is as follows. In the next sections, we describe the data being used, before demonstrating the model governing equations. Results on the reconstructed field, influence of climate variability, stability of estimation, and robustness of model are discussed thereafter. The conclusion is summarized in the last section.

\section{Improved dynamic control system model}

The model is expressed as a set of two first-order ordinary differential equations where state variables (the global mean sea level and the global mean surface temperature changes) are linearly coupled functions of time. The state variables are driven by the external nudging forcings, which generally may include those responsible for the natural variability and the anthropogenic origin. In this study, we incorporate the climate oscillations in addition to the total radiative forcing (TRF) as the nudging forcings.

To test the assumption of linear dependence of sea level and temperature on the climate variability, we compute the sea level and temperature residuals based on the DCSM model under the TRF scenario (Fig. 1). The residual of temperature is highly correlated in the multiple linear regression (MLR) with the ENSO and AMO $(r=0.55)$; a smaller coefficient $(r=0.08)$ is seen in the residual of sea level. In other words, the remaining residuals of the temperature are influenced more by the climate oscillations. As a result, we only consider the variables associated with climate variability in the model equations. Since both system comprises different physical variables with various units, we minimize the impact of scaling by the normalization. The normalization for a certain variable $R$ (denoted as $R^{*}$ ) is defined as follows:

$R^{*}=\frac{R-\bar{R}}{\max \{|R-\bar{R}|\}}$,

where $R$ is the considered variable; $\bar{R}$ is its mean value over the entire period, and $\{|R-\bar{R}|\}$ is the ensemble of absolute residual values. The set of governing equations (using normalized terms in the matrix form) are formulated as follows:

$$
\frac{d \boldsymbol{X}^{*}}{d t}=\mathbf{A} \boldsymbol{X}^{*}+\mathbf{B} \boldsymbol{Y}^{*}+\mathbf{D}
$$

where

$$
\begin{aligned}
& \boldsymbol{X}^{*}=\left[\begin{array}{c}
\boldsymbol{H}^{*} \\
\boldsymbol{T}^{*}
\end{array}\right] ; \quad \boldsymbol{Y}^{*}=\left[\begin{array}{l}
\boldsymbol{R}^{*} \\
\boldsymbol{C}_{\boldsymbol{E}}^{*} \\
\boldsymbol{C}_{\boldsymbol{A}}^{*}
\end{array}\right] ; \\
& \mathbf{A}=\left[\begin{array}{ll}
\boldsymbol{a}_{1 \boldsymbol{H}} & \boldsymbol{a}_{1 \boldsymbol{T}} \\
\boldsymbol{a}_{2 \boldsymbol{H}} & \boldsymbol{a}_{2 \boldsymbol{T}}
\end{array}\right] ; \quad \mathbf{B}=\left[\begin{array}{ccc}
0 & 0 & 0 \\
\boldsymbol{b}_{\boldsymbol{F}} & \boldsymbol{b}_{\boldsymbol{E}} & \boldsymbol{b}_{\boldsymbol{A}}
\end{array}\right] ; \quad \mathbf{D}=\left[\begin{array}{c}
\boldsymbol{d}_{\boldsymbol{H}} \\
\boldsymbol{d}_{\boldsymbol{T}}
\end{array}\right]
\end{aligned}
$$

in which $H^{*}$ and $T^{*}$ are (normalized) global mean sea level and temperature changes, respectively; $R^{*}$ (normalized) total radiative forcings, and $C_{E}^{*}$ and $C_{A}^{*}$ (normalized) climate indices representing the variability of ENSO and AMO, respectively. Here, $a_{i T}(i=1,2), a_{i H}(i=1,2), b_{F}, b_{E}, b_{A}, d_{H}$, and $d_{T}$ are constant coefficients. Matrix $\mathbf{A}$ describes the behaviors of the internal system $\mathbf{X}^{*}$, while matrix $\mathbf{B}$ characterizes the contributions from the control system $\mathbf{Y}^{*}$. Matrix $\mathbf{D}$ represents the trends. Note that, in case $b_{F}=b_{E}=b_{A}=0$, our model reduces to the DSM model of Aral et al. (2012). If further constants are eliminated, $a_{1 H}=a_{2 T}=a_{2 H}=0$; our model is simplified to the semi-empirical model by Rahmstorf (2007).

We solve the set (2) by an upwind scheme for annually average variables (i.e., $\Delta t=1$ year), as follows:

$\boldsymbol{X}_{n+1}^{*}=(\mathbf{A} \Delta t+\mathbf{I}) \boldsymbol{X}_{\boldsymbol{n}}^{*}+\mathbf{B} \boldsymbol{Y}_{n+1}^{*} \Delta t+\mathbf{D} \Delta t$,

in which $\mathbf{I}$ is the identity matrix. To determine the coefficients in Eq. (3) and estimate corresponding confidence intervals, we follow the numerical approach of Aral et al. (2012) which are implemented in detail in Appendix 1.

The estimation of parameters in Aral et al. (2012) could potentially produce overfitted solution. To resolve this issue, we adopt the tenfold cross-validation method (McLachlan et al. 2004). In this Monte Carlo technique, the dataset is randomly separated into ten equal subsets. The least squares method is then repeated ten times with nine subsets to identify the parameters in the system equations, while each subset is discarded exactly once. After that, all ten results are averaged to yield an ensemble estimation. This technique is further repeated 1000 times using the statistical bootstrapping before the mean values are determined to stably describe the system behavior.

\section{Data}

For the sea level, we use the reconstructed fields provided by Church and White (2006) which combined the altimeter and in situ observations (http://www.cmar.csiro.au/sealevel/sea level data cmar.html). With the temperature, combined land-surface air and sea-surface water temperature fields are taken from National Aeronautics and Space Administration (NASA, http://data.giss.nasa.gov/gistemp/). The annual net radiative forcing data consisting of the effect of greenhouse 


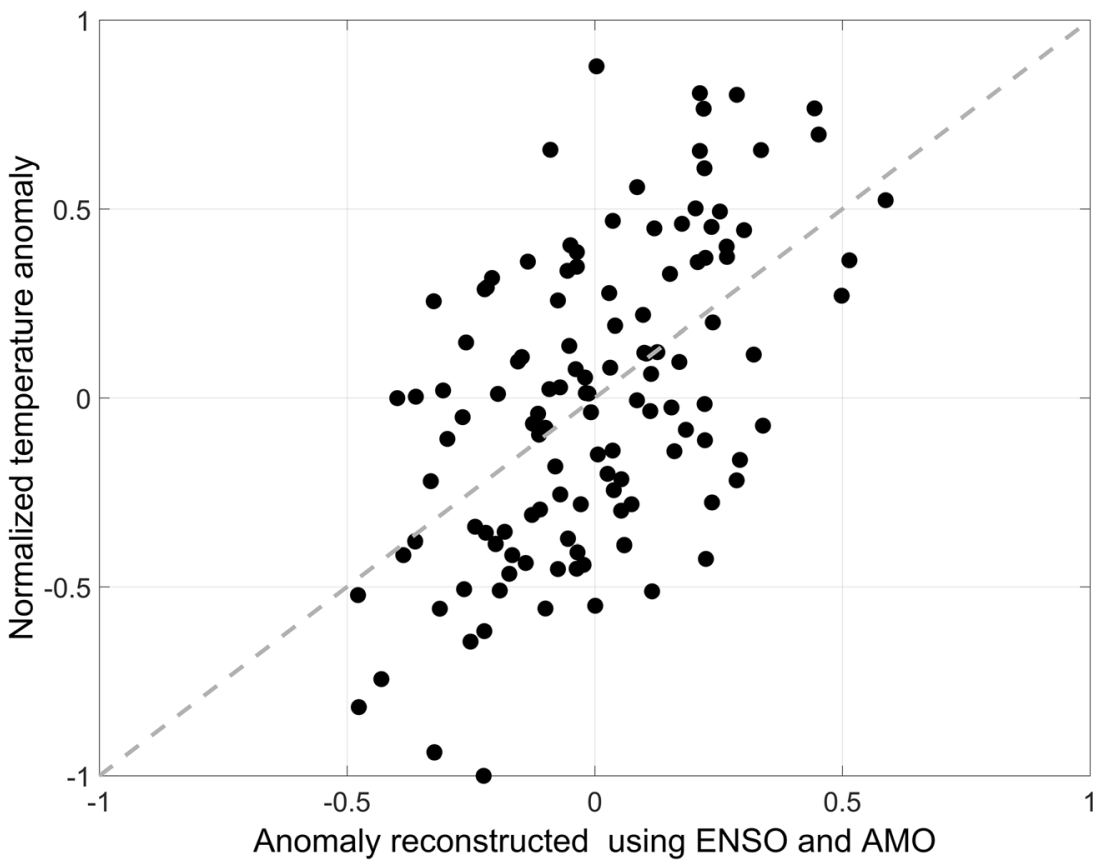

Fig. 1 Normalized temperature anomalies yielded by DCSM versus its reconstruction by multiple linear regression using AMO and ENSO climate indices. The grey dashed line is $1: 1$ reference line.

gases, human-made aerosols, volcanoes, solar irradiance, and land use are obtained from Hansen et al. (2011) at the Columbia University website (http://www.columbia. edu/ mhs119/EnergyImbalance/Imbalance.Fig01.txt). The AMO index and multivariate ENSO index (MEI) data are achieved from the National Oceanic and Atmospheric Administration (NOAA) websites (http://www.esrl.noaa.gov/psd/data/timeseries/AMO/ and http://www.esrl.noaa.gov/psd/enso/mei/, respectively). The 120-year period between 1880 and 2001 is selected for reconstruction as it is commonly available among all datasets.

\section{Results and discussions}

\subsection{Reconstructed field}

We apply our method to the historic dataset of global mean sea level and surface temperature changes with a 2 -year moving averaged smoothing as suggested by Aral et al. (2012) to minimize the random noise. We avoid using longer low-pass filters as they may distort useful information such as the climate variability.

The rates of sea level and temperature changes are modulated by the negative self-feedback with coefficients of about -0.002 and -0.305 year $^{-1}$, respectively (Table 2). For their positive interactions, surface temperature contributed to sea level rise at the rate of $2.879 \mathrm{~mm}^{\circ} \mathrm{C}^{-1}$ year $^{-1}$; while it responded to sea level change with the coefficient of $0.001 \mathrm{~mm}^{\circ} \mathrm{C}^{-1}$ year $^{-1}$. In overall, these results are well consistent with previous studies using empirical dynamic system approach (Aral et al. 2012; Guan et al. 2013). The detected TRF is positively linked to temperature change by the constant of $0.061 \mathrm{~W}^{-1} \mathrm{~m}^{2}{ }^{\circ} \mathrm{C}$. It is physically reasonable since the positive value of TRF represents more energy kept within the Earth; the ocean and atmosphere will adjust this energy imbalance, leading to the global warming and the sea level rise (whereas a negative energy budget will cause the cooling effect and sea level drop).

The reconstructions of historic evolution of global mean sea level and temperature changes from 1880 to 2001 are displayed in Fig. 2. The last two columns of Table 1 present their parameters. As shown in the figure, the DCSM produces a rational sea level reconstruction with the root mean square error (RMSE) of $7.60 \mathrm{~mm}$ and the correlation coefficient $\left(R^{2}\right)$ of 0.98 . The DCSM model performs poorer in demonstrating temperature (RMSE of $0.119{ }^{\circ} \mathrm{C}$ and $R^{2}$ of 0.76 ). DSM model also show high RMSEs for sea level $(9.63 \mathrm{~mm})$ and temperature $\left(0.122{ }^{\circ} \mathrm{C}\right)$, accompanied by a lower corresponding correlation coefficients of 0.97 and 0.74 . On the contrary, reconstructed fields from our model fit nicely with the historical data. Among the three models, our result yields the highest $R^{2}$ values of 0.98 for sea level and 0.82 for temperature. Our model is also more accurate, represented by the lowest RMSEs of $7.08 \mathrm{~mm}$ and $0.101{ }^{\circ} \mathrm{C}$ for both variables, respectively. 

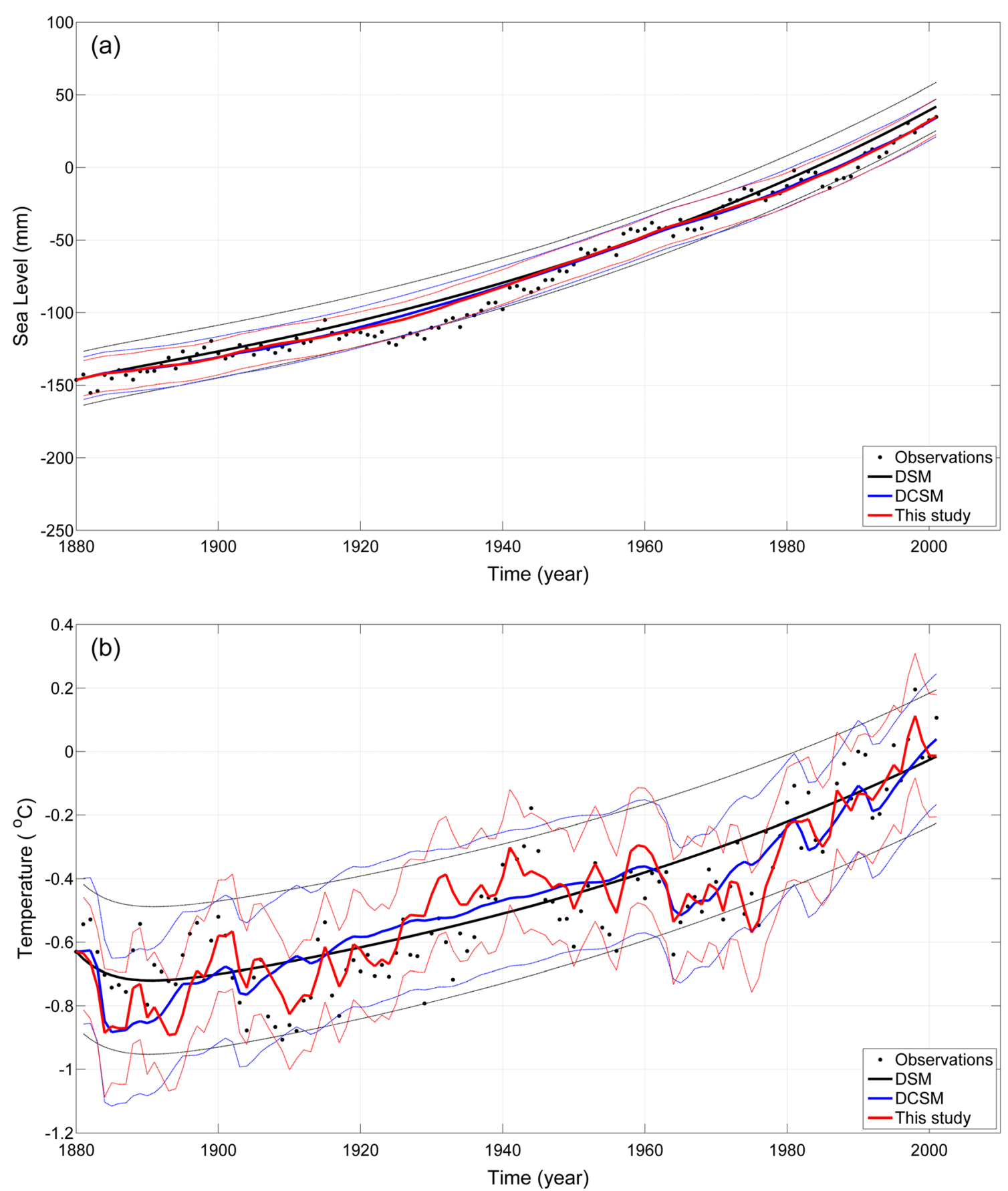

Fig. 2 Reconstruction of historic global a mean sea level and b temperature changes using different approaches from 1880 to 2001 . Lighter colored curves represent the $90 \%$ confidence intervals for corresponding thick colored curves of model reconstructions

\subsection{Influence of climate variability}

Considered the impact of external forcings as random disturbances, the simplification of Aral et al. (2012) neglected the fact that external factors may instead take part in driving changes in the system. Guan et al. (2013) shed light on the role of external controllers by showing that the inclusion of radiative forcing will improve the dynamic model. In our study, climate variability are considered as another external natural driver modulating the variability of both sea level and temperature from interannual to multi-decadal time scales. By adding ENSO and AMO climate fluctuations, the misfit around 1930 in sea level has been further improved noticeably (Fig. 2a). The most remarkable improvement is seen in the reconstruction of the global temperature change (Fig. 2b). In contrast, both DSM and DCSM can reconstruct the similar trends but their errors are much larger (Figs. 2 and 5).

The temperature change is proportional to the ENSO and AMO oscillations in a linear relationship. Their corresponding dependent constant are $0.055^{\circ} \mathrm{C}^{-1}$ year $^{-1}$ and $0.098^{\circ} \mathrm{C}^{-1}$ year $^{-1}$. 
Table 1 Parameters of three different dynamic models

\begin{tabular}{|c|c|c|c|c|c|c|c|}
\hline \multirow[t]{2}{*}{ Model } & & \multirow{2}{*}{$\begin{array}{l}\text { Internal interaction } \\
(H, T)\end{array}$} & \multicolumn{3}{|c|}{ External control variables } & \multirow[t]{2}{*}{ RMSE } & \multirow[t]{2}{*}{$R^{2}$} \\
\hline & & & $F$ & $M E I$ & $A M O$ & & \\
\hline \multirow[t]{2}{*}{ DSM } & $d H / d t$ & $\sqrt{ }$ & & & & $9.63 \mathrm{~mm}$ & 0.97 \\
\hline & $d T / d t$ & $\sqrt{ }$ & $x$ & $\times$ & $x$ & $0.122^{\circ} \mathrm{C}$ & 0.74 \\
\hline \multirow[t]{2}{*}{ DCSM } & $d H / d t$ & $\sqrt{ }$ & & & & $7.60 \mathrm{~mm}$ & 0.98 \\
\hline & $d T / d t$ & $\sqrt{ }$ & $\sqrt{ }$ & $x$ & $x$ & $0.119^{\circ} \mathrm{C}$ & 0.76 \\
\hline \multirow[t]{2}{*}{ This study } & $d H / d t$ & $\sqrt{ }$ & & & & $7.08 \mathrm{~mm}$ & 0.98 \\
\hline & $d T / d t$ & $\sqrt{ }$ & $\sqrt{ }$ & $\sqrt{ }$ & $\sqrt{ }$ & $0.101{ }^{\circ} \mathrm{C}$ & 0.82 \\
\hline
\end{tabular}

It physically implies that the positive phases of ENSO and AMO will lead to a warm effect, while the negative phases will result in the cooling down in the global surface temperature.

In estimating the global sea level rise and temperature change, Zhou and Tung (2013) indicated that past literature commonly focused muchly on considering ENSO while neglecting the AMO impact. In fact, the exclusion of AMO may cause biases in estimating global temperature trend, as being seen in the early twentieth century warming (Medhaug and Furevik 2011). By incorporating both dominant climatic factors, we reproduce the variability more accurately, in particularly, well capturing the early twentieth warming in our model (Fig. 2b).

\subsection{Stability of estimation}

The histogram of fitted coefficients is depicted in Fig. 3 and Table 2, respectively. As shown in Fig. 3, a single estimation of parameters for reconstructed field is less reliable than the ensemble as the spreading ranges of parameters are highly variant (large standard deviation). For some cases of the single estimation, coefficients lead to unrealistic physical contributions (data not shown). By increasing the number of computations through the bootstrapping technique, the best estimated coefficients is derived from its ensemble average.

The reconstructions using parameters deriving from all 1000 Monte Carlo experiments are exhibited in Fig. 4. It is found that the global mean sea level change is very sensitive to the achieved parameters in the model (Fig. 4a). The worst parameters may drive the model to excessively overshoot the reconstructed field to an unrealistic value of $200 \mathrm{~mm}$ by 2001. Instead, the best estimate gives a reconstructed sea level closed to observation by a margin of error smaller than $5 \mathrm{~mm}$ (Fig. 4a). The application of cross-validation technique in numerous Monte Carlo experiments is thus effective to lower the risk of overfitting.

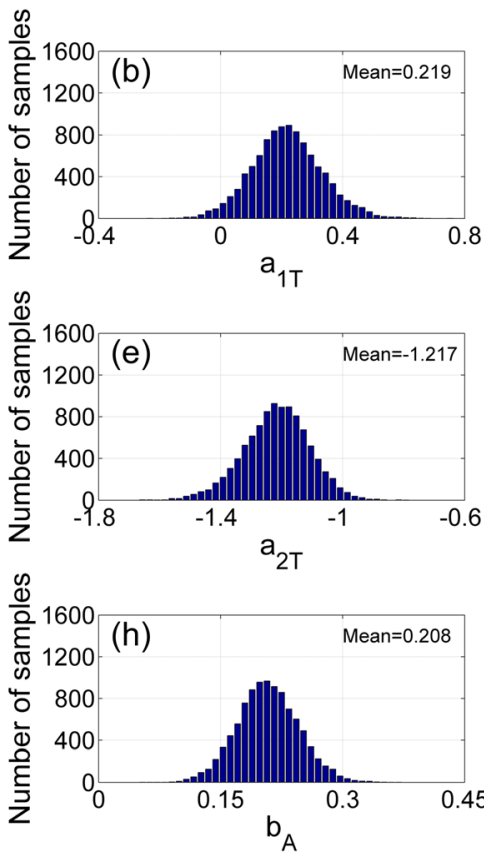
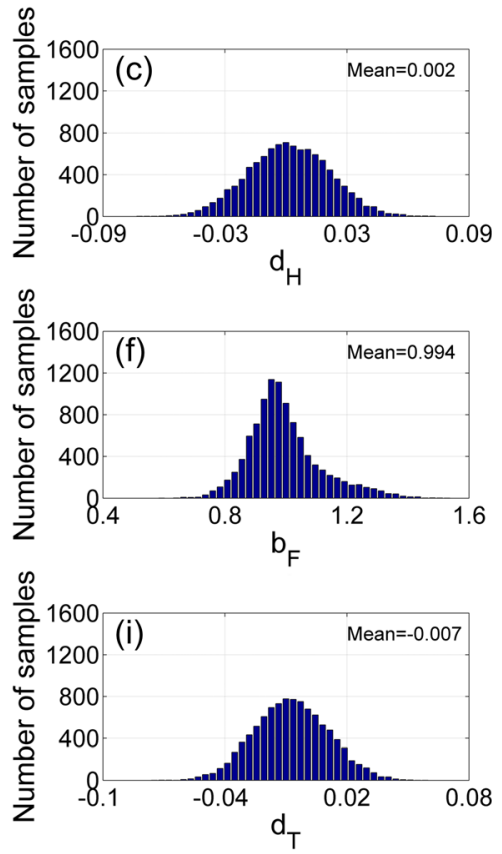

Fig. 3 a-i Histograms of the coefficients estimation deriving from 1000 Monte Carlo experiments 
Table 2 Coefficients of the system equations identified from the historic data from 1880 to 2001

Non-normalized coefficient matrixes

A

$\left[\begin{array}{cc}-0.002 & 2.879 \\ 0.001 & -0.305\end{array}\right]$

Unit matrixes of non-normalized coefficients

A

$\left[\begin{array}{cc}y r^{-1} & m m^{\circ} C^{-1} y r^{-1} \\ { }^{\circ} \mathrm{C} \mathrm{mm}^{-1} y r^{-1} & y r^{-1}\end{array}\right]$

Normalized coefficient matrixes

A

$\left[\begin{array}{cc}-0.022 & 0.219 \\ 0.399 & -1.217\end{array}\right]$
B

$\left[\begin{array}{ccc}0 & 0 & 0 \\ 0.061 & 0.055 & 0.098\end{array}\right]$

B

$\left[\begin{array}{ccc}0 & 0 & 0 \\ { }^{\circ} \mathrm{C} W^{-1} m^{2} y r^{-1} & { }^{\circ} C^{-1} y r^{-1} & { }^{\circ} C^{-1} y r^{-1}\end{array}\right]$

B

$\left[\begin{array}{ccc}0 & 0 & 0 \\ 0.994 & 0.573 & 0.208\end{array}\right]$
D

$\left[\begin{array}{c}2.788 \\ -0.109\end{array}\right]$

D

$\left[\begin{array}{l}m m y r^{-1} \\ { }^{\circ} C y r^{-1}\end{array}\right]$

D

$\left[\begin{array}{c}0.002 \\ -0.007\end{array}\right]$
As the reconstruction is executed forward, both global mean sea level and surface temperature fields anticipatively show higher uncertainties at the tails of time series, i.e., 2001. Consequently, the overshooting is more severe at the tail of reconstruction if the Monte Carlo method is not adopted. It is pointed to the fact that random sampling with replacement does increase the stability of estimation in our model.

\subsection{Robustness of model}

The accuracy of the forward reconstruction or prediction is often sensitive to the initial time, especially for the nonconstrained DSM. We recomputed the system coefficient matrices based on different time periods to examine the robustness of the model (Fig. 5). Other models are likely uncertain and less accurate in simulating both sea level and temperature changes. For example, the RMS errors in the sea level derived by the DSM model for the initial years of 1881, 1899, and 1915 are 22, 21, and $24 \mathrm{~mm}$, respectively. Meanwhile, the performance of DCSM in reconstructing historic data is slightly better, mostly due to the inclusion of TRF. For instance, the RMSEs for global temperature change reduced from an average of $0.13{ }^{\circ} \mathrm{C}$ for DSM model to $0.10^{\circ} \mathrm{C}$ for DCSM for the entire period.

In comparison with those two models, our work has a significant improvement in accuracy and robustness. The RMSE in our reconstructed temperature is reduced to $0.08{ }^{\circ} \mathrm{C}$ (Fig. 5a). The errors associated with sea level also are lessened by $10 \mathrm{~mm}$ in sea level (Fig. 5b). It suggested that our model is more vigorous and able to capture well the changes regardless of initial times. Adopting the smoothed values, reconstruction is slightly enhanced (although the role of smoothing is not crucial). The abnormal peaks in RMSEs associated with years
1899, 1915, 1953, and 1957 disappear. For the entire time series, the reconstruction derived from our model showed a better performance against other dynamic models for all periods starting in the year between 1880 and 1960 and ending in 2001. For global temperature change, our averaged root mean square errors are $24-37 \%$ smaller, and for global mean sea level rise, they are reduced by $5-25 \%$.

\section{Conclusions}

The prominent feature of the dynamic models among empirical approaches is to establish an interactive feedback mechanism between the changes of global mean sea level and global temperature. In this study, we improve its capability by introducing the terms describing natural variability. The evolutions of sea level and temperature deviations take into account two dominant and well-identified climate drivers, namely, ENSO and AMO. In comparison with other control system models (DSM and DCSM) on the same historic data from 1880 to 2001, our model showed a better performance measured by lowering RMSEs in estimating the temperature (by 17\%) and the global mean sea level (by 15\%). Correlation analysis also suggests that our reconstructed curve is closer to the historic data than other models, especially in restoring the global temperature change. For shorter periods starting in the year between 1880 and 1960 and ending in 2001, our reconstruction notably outperformed the past best estimates in both global temperature change (with 24-37\% smaller averaged RMSEs) and global mean sea level rise (with 5-25\% smaller averaged errors).

This study suggests that the direct use of climate variability information helps in improving significantly the 



Fig. 4 Reconstruction of historic global a mean sea level and $\mathbf{b}$ temperature changes. Black filled circles show the historic data; gray lines represent 1000 reconstruction experiments using the Monte Carlo method; red lines indicate our reconstruction derived from the ensemble

reconstructed fields and plausibly, the projection datasets. It is noted that the introduction of natural fluctuations are constrained by our poor predictability of climate signals, arising from our limited understanding on their physics. So far, the longest leading time of climate forecast is only about from 1 to 9 months (http://www.cpc.ncep.noaa. gov/products/CFSv2/CFSv2seasonal.shtml). Despite this, in the absence of climate variability as the control variable, our model equations are reducible to the DCSM case and, therefore, is still applicable for future projections of sea level rise and temperature changes under different emission scenarios.

There are various alternative options to improve our model. At present, the reconstructed global temperature change is better while there is only a slight enhancement in the restored global mean sea level change. At one hand, it is concluded that 

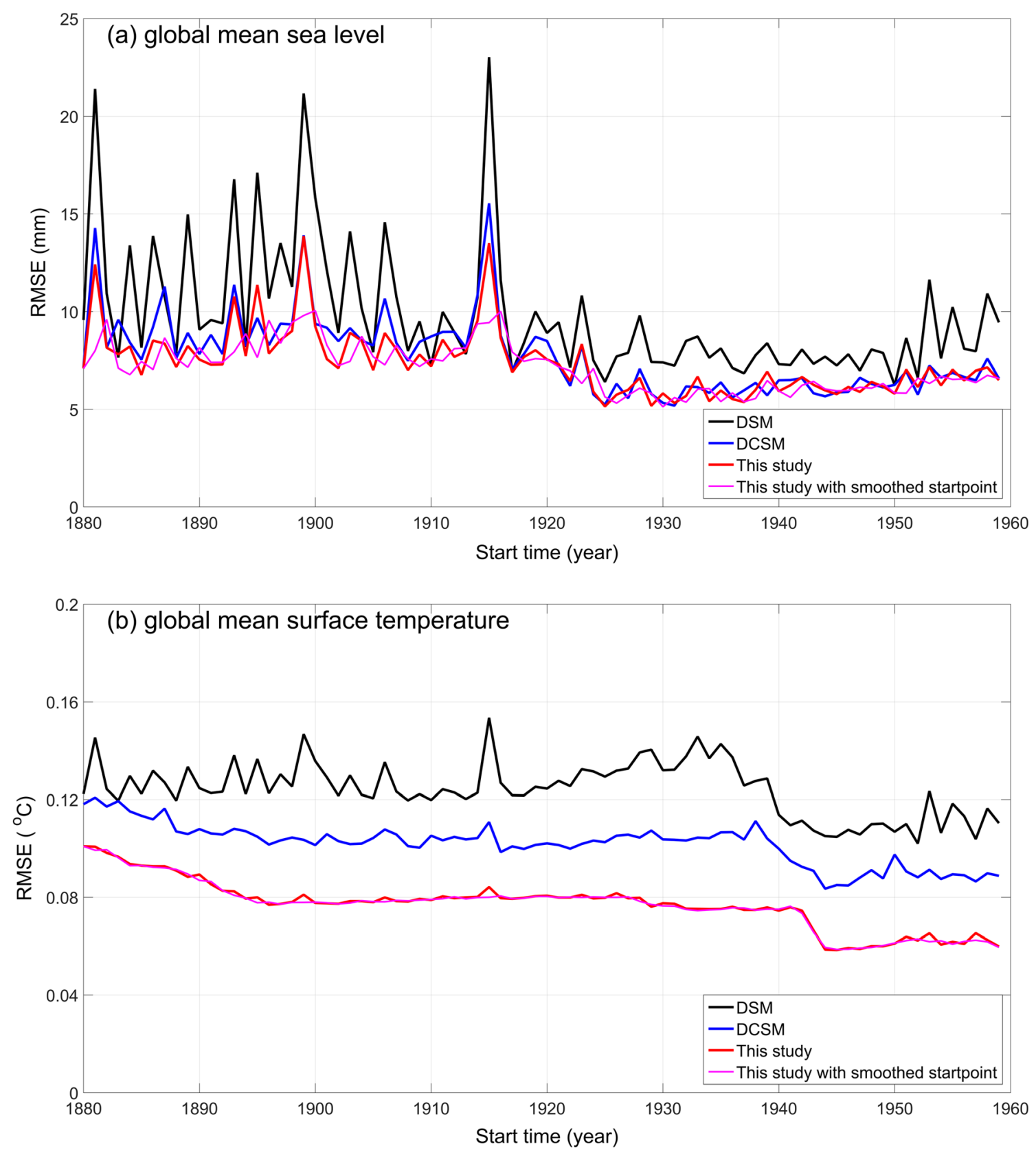

Fig. 5 Comparison of the RMSEs computed by different models for different periods initializing in a year between 1880 and 1960 and ending in the year 2001 for the changes of a global mean sea level and $\mathbf{b}$ global mean surface temperature

the influence of ENSO is non-uniform and regional, whose contribution is quantified up to $20 \mathrm{~cm}$ or more at various regions (e.g., Hamlington et al. 2015; Luu et al. 2015). On the other hand, regional sea levels are also modulated by the joint influence of AMO, the Pacific Decadal Oscillation, or the Indian Ocean Dipole (e.g., Zhang and Church 2012; Hamlington et al. 2013; Luu et al. 2015). Therefore, we may introduce the climate variability at the regional scales into a spatial temporal model. An ongoing regional analysis is being carried out to examine this approach.
A prominent advantage is that our model is less sensitive to the initial time, which is a commonly serious problem for other dynamic models. In fact, archiving the reconstructed parameters though applying a single least squares estimate could potentially contain the risk of overfitting, which will not be suitable for extending the method to the future scenarios. One may find an intermediate solution for this sensitivity through the smoothing time series (including the initial value) but the fix is inadequate. We indicate that the Monte Carlo cross-validation technique is more robust and effective to gain 
the parameters that produce more stable outputs. This bootstrapping method is thus promising in applying to improve empirical projections.

Acknowledgements This research was jointly supported by the Natural Science Foundation of China under Grant Nos. 41331172, U1406404, and 61361136001, and the Global Change and Air-Sea Interaction Project under Grant Nos. GASI-03-01-01-09. We also acknowledge the supports of the Tropical Marine Science Institute, National University of Singapore, and College of Science, Vietnam National University, Hanoi. WU Qing is a visiting student at the National University of Singapore from Ocean University of China.

\section{Appendix 1}

In this study, the system coefficients are solved by the least squares method. As abovementioned, the equations can be denoted in a simple discrete form:

$\boldsymbol{X}_{\boldsymbol{n}+1}^{*}=(\mathbf{A} \Delta t+\mathbf{I}) \boldsymbol{X}_{\boldsymbol{n}}^{*}+\mathbf{B} \boldsymbol{Y}_{\boldsymbol{n}+1}^{*} \Delta t+\mathbf{D} \Delta t$,

in which $\mathbf{X}_{n}^{*}=\left[\begin{array}{ll}H^{*}{ }_{n} & T^{*}{ }_{n}\end{array}{ }^{T}, \mathbf{Y}_{n}^{*}=\left[\begin{array}{lll}R^{*}{ }_{n} & C_{E n}^{*} & C_{A n}^{*}\end{array}\right]^{T}\right.$, and $\mathbf{I}$ is the identity matrix.

Then, based on the $N$ sets of the datasets, the equation can be further extended to be:

$\mathbf{M}_{\mathbf{i}}=\mathbf{N}_{\mathbf{i}} \mathbf{P}_{\mathbf{i}} ; \quad(\mathrm{i}=1,2)$,

where

$$
\begin{aligned}
& \mathbf{M}_{1}=\left[\begin{array}{c}
\mathbf{H}^{*}{ }_{2} \\
\mathbf{H}^{*}{ }_{3} \\
\vdots \\
\mathbf{H}^{*}{ }_{\mathrm{N}}
\end{array}\right] ; \mathbf{M}_{2}=\left[\begin{array}{c}
\mathbf{T}^{*}{ }^{2} \\
\mathbf{T}^{*}{ }^{3} \\
\vdots \\
\mathbf{T}^{*}{ }_{\mathrm{N}}
\end{array}\right] \\
& \mathbf{N}_{1}=\left[\begin{array}{ccc}
\mathbf{H}^{*}{ }_{1} & \mathbf{T}^{*}{ }_{1} & 1 \\
\mathbf{H}^{*}{ }_{2} & \mathbf{T}^{*}{ }^{2} & 1 \\
\vdots & \vdots & \vdots \\
\mathbf{H}^{*}{ }^{*}-1 & \mathbf{T}^{*}{ }^{*}-1 & 1
\end{array}\right] ; \quad \mathbf{N}_{2} \\
& =\left[\begin{array}{cccccc}
\mathbf{H}^{*} & \mathbf{T}^{*}{ }_{1} & \mathrm{R}^{*}{ }_{2} & \mathrm{C}_{\mathrm{E} 2}^{*} & \mathrm{C}_{\mathrm{A} 2}^{*} & 1 \\
\mathbf{H}^{*}{ }_{2} & \mathbf{T}^{*}{ }_{2} & \mathrm{R}^{*}{ }_{3} & \mathrm{C}_{\mathrm{E} 3} & \mathrm{C}_{\mathrm{A} 3}^{*} & 1 \\
\vdots & \vdots & \vdots & \vdots & \vdots & \vdots \\
\mathbf{H}^{*}{ }_{\mathrm{N}-1} & \mathbf{T}^{*}{ }_{\mathrm{N}-1} & \mathrm{R}^{*}{ }_{\mathrm{N}} & \mathrm{C}_{\mathrm{EN}}^{*} & \mathrm{C}_{\mathrm{AN}}^{*} & 1
\end{array}\right] ; \\
& \boldsymbol{P}_{1}=\left[\begin{array}{c}
a_{1 H} \\
a_{1 T} \\
d_{H}
\end{array}\right] . \quad \text { (12) } \boldsymbol{P}_{2}=\left[\begin{array}{c}
a_{2 H} \\
a_{2 T} \\
b_{F} \\
b_{E} \\
b_{A} \\
d_{T}
\end{array}\right] \text {. }
\end{aligned}
$$

Then, $\boldsymbol{P}_{i}$ can be estimated by the OLS estimator $\boldsymbol{P}_{i}$ :

$\mathbf{P}_{\mathbf{i}}=\left(\mathbf{N}_{\mathbf{i}}^{\mathbf{T}} \mathbf{N}_{\mathbf{i}}\right)^{-1} \mathbf{N}_{\mathbf{i}}^{\mathbf{T}} \mathbf{M}_{\mathbf{i}}$

Following Aral et al. (2012), we use the confidence intervals to describe the predicted uncertainty. Then, a $100(1$ $-\alpha) \%$ prediction confidence interval $H^{*}{ }_{C I}(i)$ and $T^{*}{ }_{C I}(i)$ at $n$th iteration, respectively, can be calculated by

$\boldsymbol{H}^{*}{ }_{\boldsymbol{C I}}(\boldsymbol{i})=\boldsymbol{Z}_{1 i} \boldsymbol{P}_{1}$

$$
\pm t_{\alpha / 2, m} \sqrt{\sigma_{T}^{2}\left(1+\boldsymbol{Z}_{1 i}\left(\boldsymbol{N}_{1}{ }^{\boldsymbol{T}} \boldsymbol{N}_{1}\right)^{-1} \boldsymbol{Z}_{1 i}^{\boldsymbol{T}}\right)},
$$

$\boldsymbol{T}^{*}{ }_{\boldsymbol{C I}}(\boldsymbol{i})=\boldsymbol{Z}_{2 i} \boldsymbol{P}_{2}$

$$
\pm t_{\alpha / 2, m} \sqrt{\sigma_{H}^{2}\left(1+\boldsymbol{Z}_{\boldsymbol{i}}\left(\boldsymbol{N}_{2}{ }^{\boldsymbol{T}} \boldsymbol{N}_{2}\right)^{-1} \boldsymbol{Z}_{2 i}^{\boldsymbol{T}}\right)},
$$

where $t_{\alpha / 2, m}$ is the value of $t$ distribution at $(1-\alpha)$ significance level and $m$ is the degrees of freedom. $\sigma_{T}^{2}$ and $\sigma_{H}^{2}$ are the variance of the normalized historic sea level and temperature data. $\hat{\boldsymbol{Z}}_{1 i}=\left[\hat{H}^{*}{ }_{i} \hat{T}^{*}{ }_{i} 1\right]$, and $\boldsymbol{Z}_{2 i}=$ $\left[\begin{array}{lll}\hat{H}^{*}{ }_{i} & \hat{T}^{*}{ }_{i} R^{*}{ }_{i+1} & C_{E i+1}^{*} \\ C_{A i+1}^{*} & 1\end{array}\right]$.

Open Access This article is distributed under the terms of the Creative Commons Attribution 4.0 International License (http:// creativecommons.org/licenses/by/4.0/), which permits unrestricted use, distribution, and reproduction in any medium, provided you give appropriate credit to the original author(s) and the source, provide a link to the Creative Commons license, and indicate if changes were made.

\section{References}

Andronova NG, Schlesinger ME (2000) Causes of global temperature changes during the 19th and 20th centuries. Geophys Res Lett 27(14):2137-2140

Aral MM, Guan J, Chang B (2012) Dynamic system model to predict global sea-level rise and temperature change. J Hydrol Eng 17(2): 237-242

Cazenave A, Dieng HB, Meyssignac B, von Schuckmann K, Decharme B, Berthier E (2014) The rate of sea-level rise. Nat Clim Chang 4: 358-361. doi:10.1038/nclimate2159

Chang B, Guan J, Aral MM (2014) Scientific discourse: climate change and sea-level rise. J Hydrol Eng 20(1):A4014003

Church JA, White NJ (2006) A 20th century acceleration in global sealevel rise. Geophys Res Lett 33(1):L01602

Church JA, White NJ (2011) Sea-level rise from the late 19th to the early 21 st century. Surv Geophys 32(4-5):585-602

Church JA, White NJ, Arblaster JM (2005) Significant decadal-scale impact of volcanic eruptions on sea level and ocean heat content. Nature 438(7064):74-77 
Dong L, Zhou T (2014) The Indian Ocean sea surface temperature warming simulated by cmip5 models during the twentieth century: competing forcing roles of GHGs and anthropogenic aerosols. J Clim 27(9):3348-3362

Falvey M, Garreaud RD (2009) Regional cooling in a warming world: recent temperature trends in the Southeast Pacific and along the west coast of subtropical South America (1979-2006). J Geophys Res 114(D4):D04102

Guan J, Chang B, Aral MM (2013) A dynamic control system model for global temperature change and sea level rise with $\mathrm{CO}_{2}$ emissions impact. Clim Res 58:55-66

Hamlington BD, Leben RR, Strassburg MW, Nerem RS, Kim K-Y (2013) Contribution of the Pacific decadal oscillation to global mean sea level trends. Geophys Res Lett 40:5171-5175. doi:10.1002 /grl.50950

Hamlington BD, Leben RR, Kim KY, Nerem RS, Atkinson LP, Thompson PR (2015) The effect of the El Nino-southern oscillation on U.S. regional and coastal sea level. J Geophys Res 120:3970-3986

Hansen J, Sato M, Kharecha P, Schuckmann KV (2011) Earth's energy imbalance and implications. Atmos Chem Phys 11(24):13421-13449

Hay CC, Morrow E, Kopp RE, Mitrovica JX (2015) Probabilistic reanalysis of twentieth-century sea-level rise. Nature 517(7535):481-484

IPCC (2007) Climate change 2007: the physical science basis. Contribution of working group I to the Fourth Assessment Report of the Intergovernmental Panel on Climate Change. Cambridge University Press, Cambridge and New York, p 996

IPCC (2013) Climate change 2013: the physical science basis. Contribution of working group I to the Fifth Assessment Report of the Intergovernmental Panel on Climate Change. Cambridge University Press, Cambridge and New York, p 1535
Jevrejeva S, Grinsted A, Moore JC (2009) Anthropogenic forcing dominates sea level rise since 1850. Geophys Res Lett 36(20):L20706

Jevrejeva S, Moore JC, Grinsted A (2012) Sea level projections to AD2500 with a new generation of climate change scenarios. Glob Planet Chang 80:14-20

Kravtsov S, Spannagle C (2008) Multidecadal climate variability in observed and modeled surface temperatures. J Clim 21(5):1104-1121

Luu QH, Tkalich P (2014) Reconstruction of gappy sea level data. Ind J Geo-Mar Sci 43(7):1316-1321

Luu QH, Tkalich P, Tay TW (2015) Sea level trend and variability around peninsular Malaysia. Ocean Sci 11:617-628. doi:10.5194/os-11617-2015

McLachlan G, Do KA, Ambroise C (2004) Analyzing microarray gene expression data. John Wiley \& Sons, Hoboken

Medhaug I, Furevik T (2011) North Atlantic 20th century multidecadal variability in coupled climate models: sea surface temperature and ocean overturning circulation. Ocean Sci 7:389-404

Rahmstorf S (2007) A semi-empirical approach to projecting future sealevel rise. Science 315(5810):368-370

Schmith T, Johansen S, Thejll P (2012) Statistical analysis of global surface air temperature and sea level using cointegration methods. J Clim 25(22):7822-7833

Trenberth KE, Fasullo JT (2013) An apparent hiatus in global warming? Earth's Futur 1(1):19-32

Vermeer M, Rahmstorf S (2009) Global sea level linked to global temperature. Proc Natl Acad Sci U S A 106(51):21527-21532

Zhang X, Church JA (2012) Sea level trends, interannual and decadal variability in the Pacific Ocean. Geophys Res Lett 39(21):L21701. doi: $10.1029 / 2012 \mathrm{gl} 1053240$

Zhou J, Tung KK (2013) Deducing multidecadal anthropogenic global warming trends using multiple regression analysis. J Atmos Sci 70(1):3-8 